J Ocular Biol

March 2014 Vol.:2, Issue:1

(C) All rights are reserved by Ascaso et al

\section{Multicenter Study of} Infectious and Noninfectious Endophthalmitis after Intravitreal Ranibizumab Injections

Keywords: Age-related macular degeneration; Anti-VEGF drugs; End ophtha Imitis; Intra vitreal injection a dverse-effects; Ra nibizumab

\begin{abstract}
Study background: The purpose of this study is to evaluate the overall incidence of infectious and non infectious endophthalmitis associated with intra vitreal ranibizumab (IVR) injections.

Methods: This is a retrospective, consecutive, multic enter case series involving six large clinical sites. The number of injections was determined from the injection log books and billing records. Cases of clinical diagnoses of endophthalmitis or suspected endophthalmitis resulting from IVR injection were identified and reviewed. From these data, the risk per injection was estimated.
\end{abstract}

Results: A total of 7,178 IVR injections were administered on 1,372 patientsfrom J a nuary 1, 2007 to J uly 31,2010 . In thisseries, the inc idence of clinic ally-suspected and culture-positive infectious end ophtha Imitis was 3 cases for total number of 7,178 IVR injections (0.042\%), whereas the incidence of clinically-suspected and culture-negative infectious end ophtha Imitis was only 1 case $(0.014 \%)$, and the incidence of sterile noninfectious endophthalmitis was also 1 case $(0.014 \%)$. The clinica course was favorable in all patients. Posteriorly, three of the patients received other IVR injections with no complic ations.

Conclusions: The rate of c ulture-proven end ophtha Imitisa ssoc ia ted with IVR injections is low, with an incidence of approximately 1 in 2,392 injections. The prognosis is good and patients with this complic ation do well, typic ally with a retum to baseline vision.

\section{Introduction}

The intravitreal use of anti-vascular endothelial growth factor (anti-VEGF) agents has become the standard of care for the treatment of neovascular age-related macular degeneration (AMD). In particular, intravitreal ranibizumab (IVR) has been demonstrated to be very efficacious in different well-designed clinical trials $[1,2]$. It is also increasingly being used to treat other types of choroidal neovascularization (CNV) and macular edema of various etiologies [39]. Additionally, patients often require multiple periodic reinjections to achieve or maintain a therapeutic effect. Given both the widespread use of IVR and the potential need for frequent reinjections, the safety of these injections continues to be scrutinized.

Ranibizumab (Lucentis, Genentech Inc., South San Francisco, $\mathrm{CA}$ ) is a fragment of a humanized, monoclonal antibody that binds to all VEGF-A isoforms, thereby, inhibiting angiogenesis and reducing vascular permeability [10]. It was specifically designed for ophthalmic use and was approved by the U.S. Food and Drug Administration for the treatment of wet AMD in June 2006. It has also shown great

\section{Journal of}

\section{Ocular Biology}

Francisco J. Ascaso ${ }^{1,7 *}$, Luis Arias $^{2}$, José L. Olea $^{3}$, Laura Villén ${ }^{1}$, Marta S. Figueroa ${ }^{4}$, Eduardo Esteban $^{5}$ and Alfredo García-Layana ${ }^{6}$

${ }^{1}$ Department of Ophthalmology, "Lozano Blesa" University Clinic Hospital, Zaragoza, Spain

${ }^{2}$ Department of Ophthalmology, Bellvitge University Hospital, L'Hospitalet de Llobregat, Barcelona, Spain

${ }^{3}$ Department of Ophthalmology, "Son Dureta" University Hospital, Palma de Mallorca, Spain

${ }^{4}$ Department of Ophthalmology, "Ramón y Cajal" University Hospital, Madrid, Spain

${ }^{5}$ Department of Ophthalmology, "Virgen Macarena" University Hospital, Sevilla, Spain

${ }^{6}$ Department of Ophthalmology, University Clinic of Navarra, Pamplona, Spain

${ }^{7}$ Department of Ophthalmology, Aragon Health Sciences Institute, Zaragoza, Spain

*Address for Correspondence

Francisco J. Ascaso, MD, PhD, Department of Ophthalmology, University Clinic Hospital, San Juan Bosco 15, ES-50009 Zaragoza, Spain, Tel: + 34 686574389; Fax: + 34 976565995; Email: jascaso@gmail.com

Submission: 22 February 2014

Accepted: 06 March 2014

Published: 11 March 2014

Reviewed \& Approved by: Dr. Eric W Hein, Department of Ophthalmology, Wake Forest Baptist Health Eye Center, USA

efficacy in the management of macular edema attributable to a variety of causes $[1,2]$.

Endophthalmitis is the most feared complication of intravitreal injection of anti-VEGF drugs in general $[1,11,12]$ and in particular ranibizumab [13,14]. Postinjection inflammation may represent culture-positive infectious endophthalmitis, culture-negative infectious endophthalmitis, or sterile noninfectious inflammation [15]. Culture-positive infectious endophthalmitis produces a potential loss of vision, which can be permanent in spite of prompt and appropriate management. The reported incidence of culture-positive infectious endophthalmitis is $0 \%$ to $0.007 \%$ after IVR injection $[1,11$. Some cases of culture-negative endophthalmitis are truly infectious, but the inciting organism may be too difficult to grow in culture, and some cases are treated empirically with intravitreal antibiotics without performing a culture to prove an infectious etiology $[1,13]$. Other cases of culture-negative endophthalmitis resolve without intravitreal antibiotics and represent sterile noninfectious inflammation; the reported incidence of these cases for IVR injections is $0.02 \%$ to $0.07 \%$ $[1,16]$. The aim of the present multicenter study was to evaluate outcomes of endophthalmitis after IVR injection.

\section{Material and Methods}

This study was a multicenter, retrospective, nonrandomized interventional consecutive case series. Inclusion criteria incorporated all patients underwent IVR injections in the Departments of Ophthalmology at Bellvitge University Hospital (Barcelona, Spain), "Son Dureta" University Hospital (Palma de Mallorca, Spain), "Lozano Blesa" University Clinic Hospital (Zaragoza, Spain), "Ramon y Cajal" University Hospital (Madrid, Spain), University Clinic of Navarra (Pamplona, Spain), and "Virgen Macarena" University Hospital (Sevilla, Spain) from January 1, 2007 to July 31, 2010. The study and data accumulation were performed with the approval of the 
Citation: Ascaso FJ, Arias L, Olea JL, Villén L, Figueroa MS, et al. Multicenter Study of Infectious and Noninfectious Endophthalmitis after Intravitreal Ranibizumab Injections. J Ocular Biol. 2014;2(1): 5.

local ethics committee of each hospital. Injections were recorded in nursing log books and these were later reviewed to assess total number of injections of this vascular targeting agent. Clinical indications for injection included: neovascular AMD, myopic CNV, and macular edema associated with various conditions. All participating patients gave informed consent. We obtained Institutional Review Board approval before initiating the study at each institution.

Patients were treated with IVR injections $(0.5 \mathrm{mg} / 0.05 \mathrm{~mL})$ in the surgery room under sterile conditions following the protocol for intravitreal injections of the Vitreous-Retina Spanish Society (SERV). The surface of the eye was anesthetized by applying a commercial preparation containing tetracaine and oxybuprocaine ("Colircusi Anestésico Doble," Laboratorios Alconcusí, El Masnou, Spain). High-viscosity preserved tetracaine hydrochloride 0.5\% (Cynacon/ Ocusoft, Rosenberg, Texas, USA) drops were instilled and were left on the surface of the eyes for approximately 5 minutes. The eyelids and eyelashes were prepared with $10 \%$ povidone-iodine. The ocular surface was disinfected with $5 \%$ povidone-iodine from single-use dispensers into each conjunctiva. We used a sterile wire lid speculum, sterile gloves, and a surgical drape. Attention was paid not to touch the tip of any instruments. The injection was performed in the inferior temporal quadrant $3.5 \mathrm{~mm}$ to $4.0 \mathrm{~mm}$ posterior to the limbus with a 30 -gauge needle. The needle was withdrawn from the eye, the injection site was compressed with a cotton swab to avoid reflux, and additional drops of tobramycin and 5\% povidone-iodine were placed. The patients were given a bottle of tobramycin eye drops at no charge to use four times per day for four days following the injection. They were encouraged to call us if they had any problems. All patients were rigorously evaluated before the procedure and follow-up examinations were carried out every 4 weeks.

Suspected endophthalmitis was defined as an intraocular inflammation of any amount leading to sufficient suspicion of infection that the patient was given intravitreal antibiotics with a vitreous tap or systemic antibiotics. Signs of infection included marked fibrin, intraocular inflammation, pain, and/or significant vision loss presenting within a few days of injection. Cases of postinjection uveitis that were not clinically suspected of infection were excluded. A vitreous specimen of the suspected cases was sent to the Microbiology laboratory of each hospital for gram stain, fungal, aerobic and anaerobic cultures. Proven endophthalmitis was considered to be present if the patient had either a positive gram stain or culture.

\section{Results}

A total of 7,178 IVR injections were administered on 1,372 patients from January 1, 2007 until July 31, 2010. The average number of injections per patient per eye was 5.2, with a median number of 2 and a range of 1 to 16 injections per patient. The mean time of postinjection follow-up was 39 days. Analysis of all injections documented only five reported cases of clinically-suspected endophthalmitis following injection with ranibizumab (Table 1).

In this series, the incidence of clinically-suspected and culturepositive infectious endophthalmitis was 3 cases for total number of 7,178 IVR injections $(0.042 \%)$, whereas the incidence of clinically-

Table 1: Main data of patients suffering endophthalmitis following intravitreal ranibizumab injection.

\begin{tabular}{|c|c|c|c|c|c|}
\hline Case no. & 1 & 2 & 3 & 4 & 5 \\
\hline Sex & $\mathrm{F}$ & $M$ & M & $\mathrm{F}$ & $\mathrm{F}$ \\
\hline Age (years) & 65 & 68 & 61 & 77 & 65 \\
\hline Diagnosis & AMD & AMD & AMD & AMD & AMD \\
\hline Location & Juxtafoveal CNV & Juxtafoveal CNV & Subfoveal CNV & Subfoveal CNV & Juxtafoveal CNV \\
\hline Injection no. & $3^{\text {rd }}$ & $4^{\text {th }}$ & $2^{\text {nd }}$ & $1^{\text {st }}$ & $3^{\text {rd }}$ \\
\hline Days after injection & 3 & 2 & 2 & 5 & 3 \\
\hline Visual acuity loss & + & + & + & + & + \\
\hline Ocular pain & + & + & - & + & + \\
\hline Red eye & + & + & + & + & + \\
\hline Hypopyon & + & + & + & + & + \\
\hline Vitritis & + & + & + & + & + \\
\hline Vitreous culture & S. epidermidis & S. aureus & $(-)$ & Neisseria sp & $(-)$ \\
\hline Treatment & $\begin{array}{l}\text { Intravitreal } \\
\text { antibiotics }\end{array}$ & $\begin{array}{l}\text { Intravitreal antibiotics } \\
+ \text { vitrectomy }\end{array}$ & $\begin{array}{c}\text { Periocular } \\
\text { corticosteroids }\end{array}$ & $\begin{array}{l}\text { Intravitreal } \\
\text { antibiotics }\end{array}$ & $\begin{array}{l}\text { Intravitreal } \\
\text { antibiotics }\end{array}$ \\
\hline Diagnosis & $\begin{array}{l}\text { Culture + infectious } \\
\text { endophthalmitis }\end{array}$ & $\begin{array}{c}\text { Culture + infectious } \\
\text { endophthalmitis }\end{array}$ & $\begin{array}{c}\text { Sterile noninfectious } \\
\text { endophthalmitis }\end{array}$ & $\begin{array}{c}\text { Culture + infectious } \\
\text { endophthalmitis }\end{array}$ & $\begin{array}{c}\text { Culture - infectious } \\
\text { endophthalmitis }\end{array}$ \\
\hline
\end{tabular}

Baseline

BCVA

$\mathrm{HM}$

CF

LP

$\mathrm{HM}$

CF

Post-treatment 
Citation: Ascaso FJ, Arias L, Olea JL, Villén L, Figueroa MS, et al. Multicenter Study of Infectious and Noninfectious Endophthalmitis after Intravitreal Ranibizumab Injections. J Ocular Biol. 2014;2(1): 5.

suspected and culture-negative infectious endophthalmitis was only 1 case $(0.014 \%)$, and the incidence of sterile noninfectious endophthalmitis was also 1 case $(0.014 \%)$.

\section{Discussion}

Today, antiangiogenic therapy is the standard treatment for CNV secondary to AMD. In particular, IVR injections have been demonstrated to be very efficacious in different well-designed clinical trials $[1,2]$. Likewise, they are also increasingly employed to treat other types of CNV and macular edema of various etiologies [3-9].

The risk of endophthalmitis after IVR injection is low. However, it must be explained to the patient and assumed before starting treatment. In MARINA study, presumed endophthalmitis occurred in five of 477 patients (rate per patient of 1\%) who received a total of 10,443 IVR injections (rate per injection of $0.05 \%$ ) [1]. In the ANCHOR study, during the first 12 months [2], presumed endophthalmitis occurred in two (1.4\%) patients receiving ranibizumab $0.5 \mathrm{mg}$, one of whom had serious uveitis treated with antibiotics. In the PIER study, patients received ranibizumab 0.3 or $0.5 \mathrm{mg}$ or a sham injection at monthly intervals for 3 months, followed by fixed quarterly dosing [17]. This regimen was generally well tolerated and there were no reported cases of endophthalmitis or serious uveitis. Pilli et al. [13], among 6,347 ranibizumab injections, found two cases of suspected endophthalmitis (rate per injection of $0.031 \%$ ); these cases could not be culture-proven and both patients regained their preinjection visual acuity. Fintak et al. [11] reported three infectious endophthalmitis after administration of a total of 14,320 injections of IVR. Two of these patients were culture positive. The rate of endophthalmitis associated with IVR was low, with an incidence of approximately 1 in 4,500 injections $(0.02 \%)$. In the 1 -year safety results from SAILOR study [18], where 2,378 patients each received an average number of injections of 4.6, the incidence of ocular inflammation classified as adverse events (including iritis, uveitis, vitritis and iridocyclitis) was 1.0 and $1.5 \%$ for the 0.3 and $0.5 \mathrm{mg}$ treatment groups, respectively. Bhavsar et al. [19] reported the incidence of endophthalmitis after IVR injection by means of a standardized procedure that does not require topical antibiotics, sterile gloves, or a sterile drape. Three cases of culture-positive endophthalmitis occurred after IVR injections (0.09\%). In all 3 cases of endophthalmitis, topical antibiotics were given for several days after the injection but not before injection. Lima et al. [20] evaluated the safety for bilateral same-day intravitreal injections of anti-VEGF therapy. The incidence of culture-proven endophthalmitis was $0.065 \%$, and the incidence of acute intraocular inflammation was $0.033 \%$. The complication rates after bilateral same-day intravitreal anti-VEG injections seem to be similar to those after unilateral injections. Klein et al. [8] examined 22,579 IVR injections. They identified 10 cases of presumed endophthalmitis $(0.044 \%)$. Eight of these 10 cases were diagnosed within 4 days of the intravitreal injection. Mean time to diagnosis was 3.5 days. Three of $10(46 \%)$ cases were culture positive. Culture data were not available for 2 of the cases. Only gram positive organisms were isolated: Staphylococcus epidermidis $\times 2$, coagulase-negative staphylococcus $\times 1$ (not speciated). Four of the 10 patients returned to baseline vision ( \pm 1 line). During the 24 month follow-up of a single-center, prospective study of 138 cases treated with ranibizumab $0.5 \mathrm{mg}$ there were no cases of endophthalmitis [21]. A retrospective analysis of
14,320 IVR injections revealed an incidence of endophthalmitis of $0.02 \%$ ( 1 in 4,500 injections) [22], which is broadly consistent with the rate of $0.05 \%$ per injection seen in MARINA study [11] and $<0.1 \%$ per injection seen in ANCHOR study [16]. Two recent retrospective reviews of 725 and 981 injections of ranibizumab, respectively, revealed no incidences of endophthalmitis $[22,23]$. In a multicenter, randomized clinical trial the Diabetic Retinopathy Clinical Network [24] reported 3 injection-related cases of infectious endophthalmitis ( 1 after a study injection at baseline, 1 after an injection at 4 weeks, and 1 after an injection at 56 weeks) following the 3,973 IVR injections $(0.08 \%)$ among 375 study participants $(0.8 \%)$. Ness et al. [25] reported 11 patients with severe intraocular inflammation after intravitreal injection. Only one of them was infectious endophthalmitis with retinal abscess. All others were toxic vitreitis. Seven eyes exhibited hypopyon and five disseminated retinal hemorrhages. The toxic reaction occurred within 48 hours after injection, whereas in the endophthalmitis case, it occurred after 72 hours. They believed that the cause of this reaction was the particular syringe brand used. After changing to another syringe brand, no further cases of toxic vitreitis occurred during the next 6 months. Toxic inflammatory reaction is not only a complication of cataract surgery, but may also occur after intravitreal injection [25]. In the series of Fong et al. [26], the frequency of endophthalmitis was $0.3 \%$ (2/648 injections). Recently, Rasmussen et al. [27], in a 4-year longitudinal study of 555 patients treated with ranibizumab for neovascular $\mathrm{AMD}$, found that endophthalmitis occurred in two eyes of 7,584 injections (0.026\%). Likewise, in a longterm outcomes of ranibizumab therapy for diabetic macular edema, the RIDE and RISE Research group found that per-injection rates of endophthalmitis remained low over time (0.06\% per injection) [28].

In our study, the incidence of culture-positive infectious endophthalmitis following IVR injection was $0.042 \%(3 / 7,178$ injections). Similar incidences of presumed endophthalmitis (0.03 $0.077 \%$ ) have been reported in other recent retrospective studies of intravitreal anti-VEGF agents including ranibizumab [13,29,30]. In the largest consecutive case review series to date (Timothy Murray, communication with AAM), 34,278 patients from the Bascom Palmer Eye Institute and its satellite clinics undergoing treatment with intravitreal anti-VEGF drugs were reviewed between 2005 and 2008 , and the per-injection rate of endophthalmitis was found to be $0.039 \%$ after IVR injections.

The pathogens most commonly isolated from intravitreal cultures are Staphylococcus epidermidis and coagulase-negative staphylococci $[8,22,26,29]$. Likewise, species of the Staphylococcus genus were isolated in our cases no. 1 and 2 (S. Epidermidis and $S$. Aureus, respectively). Both cases responded well to treatment and the clinical course was favorable with anatomic and visual improvement. Posteriorly, they received other IVR injections with no complications. Neisseria sp. was isolated in our case no. 4, resulting in anatomic resolution but no visual improvement.

The ocular surface bacteria at the time of injection are the likely source of bacteria for postinjection infection [31]. The theoretical likelihood of having high enough concentration of viable bacteria remaining in the volume of tear film potentially displaced into the eye after disinfection by topical application of povidone-iodine to cause endophthalmitis appears to be very low. This seems to be borne 
Citation: Ascaso FJ, Arias L, Olea JL, Villén L, Figueroa MS, et al. Multicenter Study of Infectious and Noninfectious Endophthalmitis after Intravitreal Ranibizumab Injections. J Ocular Biol. 2014;2(1): 5.

out in our results, in that the incidence of suspected and proven endophthalmitis in our patients was very infrequent.

Difficulty remains in clinically differentiating infectious endophthalmitis from noninfectious endophthalmitis, as there is great overlap between these two entities. In infectious endophthalmitis there is typically an anterior chamber cellular reaction with at least some degree of fibrin, which is associated with pain and scleral or conjunctival inflammation [32,33]. Sterile noninfectious endophthalmitis can present with a hypopyon that may shift with changes in head position, inflammation that is usually only mild and self-limited, and typically there is no pain. As we observed in our case no. 3 , the symptoms use to quickly improve with topical or systemic corticosteroids [32,33]. In the present study, the incidence of sterile noninfectious endophthalmitis was only 1 case for total number of 7,178 injections $(0.014 \%)$. The reported incidence of these cases of noninfectious sterile endophthalmitis for IVR injections is $0.02 \%$ to $0.07 \%$, and its etiology is not known $[1,16]$. Some believe that it is attributable to the preservative, or to endotoxin, but it has been reported after compounded preservative-free triamcinolone [33], and no endotoxin has been found. It is possible that the inflammation may be caused by something in the syringe, needles, or surface of the eye (e.g., povidone-iodine) tracking into the eye with the injection, but not the drug itself [33].

The present study has some obvious shortcomings that should be recognized. It is a retrospective study, nonrandomized, with six participating centers, and without a control group. Its strengths lie on the large number of injections analyzed and the fact that one hundred percent of the injected patients received standardized evaluation and treatment according to the same protocol for intravitreal injections of the Vitreous-Retina Spanish Society (SERV) and with certified technicians to perform the examinations. Moreover, all patients were seen on a follow-up visit four weeks later, so there was no loss to follow-up.

Is there benefit for a theater setting? Although it has been believed that use of a filtered air environment decreases the risk of infection by airborne pathogens, a high percentage of injections take place in the physician's office and the documented low rates of endophthalmitis support the safety of this procedure [12,32]. Additional pros of inoffice setting would be cost-effectiveness reasons such as time, expense and facility requirements. Nevertheless, some ophthalmologists are incentivized to perform injections in the operating theater for reasons of reimbursement, and in a single surgeon comparative cohort study in-theatre intravitreal injections were associated with a 13 fold lower risk of endophthalmitis compared to in-office injections [34]. It is difficult to determine whether specific infection precaution measures, particularly the use of drapes, sterile gloves, and prepping of periocular skin have any impact on actual rates of infection [29]. Suggested standardized injection protocols based on expert consensus agree on the use of a lid speculum, preoperative povidone-iodine, appropriate anesthetic, and avoidance of extensive massage to the eyelids $[29,32,33]$. We draw attention to the use of povidone-iodine, which is considered level 2 evidence in expert opinion. Its use has been associated with a reduced number of colonies and a confirmed lower risk of post-injection infections [35]. In a trial conducted by Speaker et al. [31] where the application of povidone-iodine to the ocular surface before surgery was compared to the preoperative use of silver solution, a significantly lower incidence of culture-positive endophthalmitis was observed postoperatively in cases where povidone-iodine was utilized prophylactically. This evidence further corroborates the use of povidone-iodine, applied directly to the ocular surface, eyelid margins, and eye lashes before any intravitreous injection [31]. Another important part of the peri-injection protocol is the lid speculum. It is recommended to avoid needle contact with lids and lashes at all times, but its sterility is not mandatory [31]. Furthermore, Fineman et al. [36] has recently described an alternative technique for avoiding contact with the lids and eyelashes without the use of a metal lid speculum along with the results in clinical practice. This technique of bimanual assisted eyelid retraction for intravitreal injection has a low rate of infection similar to the reported rates using a metal lid speculum.

Controversy has revolved around the role of topical antibiotics in the prevention of endophthalmitis, but it is still unclear whether these agents are of any benefit [37]. The rate of endophthalmitis does not seem to be higher when topical antibiotics were not used before, on the day of, or after intravitreal injections [19,38,39]. Despite this debate, it is customary in our services to use post-injection tobramicin prophylactically, due to evidence showing that topical antibiotics lower the number of bacteria cultured from the conjunctiva and lids [40]. Tobramicin is a low-cost, well tolerated antibiotic, which shows a good sensitivity profile against both gram-positive and negative organisms. There is no apparent increase of coagulase-negative staphylococci resistance to tobramicin, as has previously been observed with fluoroquinolones [41].

In conclusion, the rate of culture-proven infectious endophthalmitis associated with IVR injections is low, with an incidence of approximately 1 in 2,392 injections. Patients with this complication do well, typically with a return to baseline vision. We highlight the importance of IVR injections performed with standardized sterile techniques and close follow-up of patients as major contributing factors to such a low occurrence.

\section{References}

1. Rosenfeld PJ, Brown DM, Heier JS, Boyer DS, Kaiser PK, et al. (2006) Ranibizumab for neovascular age-related macular degeneration. $\mathrm{N}$ Engl J Med 355: 1419-1431.

2. Brown DM, Kaiser PK, Michels M, Soubrane G, Heier JS, et al. (2006) Ranibizumab versus verteporfin for neovascular age-related macular degeneration. N Engl J Med 355: 1432-1444.

3. Peyman GA, Lad EM, Moshfeghi DM (2009) Intravitreal injection of therapeutic agents. Retina 29: 875-912.

4. Stepien KE, Eaton AM, Jaffe GJ, Davis JL, Raja J, et al. (2009) Increased incidence of sterile endophthalmitis after intravitreal triamcinolone acetonide in spring 2006. Retina 29: 207-213.

5. Rosenfeld PJ, Moshfeghi AA, Puliafito CA (2005) Optical coherence tomography findings after an intravitreal injection of bevacizumab (avastin) for neovascular age-related macular degeneration. Ophthalmic Surg Lasers Imaging 36: 331-335.

6. Greenberg PB, Martidis A, Rogers AH, Duker JS, Reichel E (2002) Intravitreal triamcinolone for the treatment of macular oedema due to central retinal vein occlusion. Br J Ophthalmol. 86: 247-248.

7. Heier JS, Antoszyk AN, Pavan PR, Leff SR, Rosenfeld PJ, et al. (2006) Ranibizumab for treatment of neovascular age-related macular degeneration: a phase I/II multicenter, controlled, multidose study. Ophthalmology 113: 633. 
Citation: Ascaso FJ, Arias L, Olea JL, Villén L, Figueroa MS, et al. Multicenter Study of Infectious and Noninfectious Endophthalmitis after Intravitreal Ranibizumab Injections. J Ocular Biol. 2014;2(1): 5.

8. Klein KS, Walsh MK, Hassan TS, Halperin LS, Castellarin AA, et al. (2009) Endophthalmitis after anti-VEGF injections. Ophthalmology 116: 1225.

9. Fung AE, Palanki R, Bakri SJ, Depperschmidt E, Gibson A (2009) Applying the CONSORT and STROBE statements to evaluate the reporting quality of neovascular age-related macular degeneration studies. Ophthalmology 116 286-296.

10. Gaudreault J, Fei D, Rusit J, Suboc P, Shiu V (2005) Preclinica pharmacokinetics of Ranibizumab (rhuFabV2) after a single intravitrea administration. Invest Ophthalmol Vis Sci 46: 726-733.

11. Fintak DR, Shah GK, Blinder KJ, Regillo CD, Pollack J, et al. (2008) Incidence of endophthalmitis related to intravitreal injection of bevacizumab and ranibizumab. Retina 28: 1395-1399.

12. Jager RD, Aiello LP, Patel SC, Cunningham ET Jr (2004) Risks of intravitreous injection: a comprehensive review. Retina 24: 676-698.

13. Pilli S, Kotsolis A, Spaide RF, Slakter J, Freund KB, et al. (2008) Endophthalmitis associated with intravitreal anti-vascular endothelial growth factor therapy injections in an office setting. Am J Ophthalmol 145: 879-882.

14. Mason JO 3rd, White MF, Feist RM, Thomley ML, Albert MA, et al. (2008) Incidence of acute onset endophthalmitis following intravitreal bevacizumab (Avastin) injection. Retina 28: 564-567.

15. Chong DY, Anand R, Williams PD, Qureshi JA, Callanan DG (2010) Characterization of sterile intraocular inflammatory responses after intravitreal bevacizumab injection. Retina 30: 1432-1440.

16. Brown DM, Michels M, Kaiser PK, Heier JS, Sy JP, et al. (2009) Ranibizumab versus verteporfin photodynamic therapy for neovascular age-related macula degeneration: two-year results of the ANCHOR study. Ophthalmology I16: 57-65

17. Regillo CD, Brown DM, Abraham P, Yue H, lanchulev T, et al. (2008) Randomized, double-masked, sham-controlled trial of ranibizumab for neovascular age-related macular degeneration: PIER study year 1 . Am J Ophthalmol 145: 239-248.

18. Boyer DS, Heier JS, Brown DM, Francom SF, Ianchulev T, et al. (2009) A Phase IIIb study to evaluate the safety of ranibizumab in subjects with neovascular age-related macular degeneration. Ophthalmology 116: 17311739.

19. Bhavsar AR, Googe JM Jr, Stockdale CR, Bressler NM, Brucker AJ, et al. (2009) Risk of endophthalmitis after intravitreal drug injection when topical antibiotics are not required: the diabetic retinopathy clinical research network laser-ranibizumab-triamcinolone clinical trials. Arch Ophthalmol 127: 15811583.

20. Lima LH, Zweifel SA, Engelbert M, Sorenson JA, Slakter JS, et al. (2009) Evaluation of safety for bilateral same-day intravitreal injections of antivascular endothelial growth factor therapy. Retina 29: 1213-1217.

21. Rothenbuehler SP, Waeber D, Brinkmann CK, Wolf S, Wolf-Schnurrbusch UE (2009) Effects of ranibizumab in patients with subfoveal choroidal neovascularization attributable to age-related macular degeneration. Am J Ophthalmol 147: 831-837.

22. Ladas ID, Karagiannis DA, Rouvas AA, Kotsolis Al, Liotsou A, et al. (2009) Safety of repeat intravitreal injections of bevacizumab versus ranibizumab: our experience after 2,000 injections. Retina 29: 313-318.

23. Cavalcante LL, Cavalcante ML, Murray TG, Vigoda MM, Piña Y, et al. (2010) Intravitreal injection analysis at the Bascom Palmer Eye Institute: evaluation of clinical indications for the treatment and incidence rates of endophthalmitis. Clin Ophthalmol 4: 19-524.
24. Diabetic Retinopathy Clinical Research Network, Elman MJ, Aiello LP, Beck RW, Bressler NM, et al. (2010) Randomized trial evaluating ranibizumab plus prompt or deferred laser or triamcinolone plus prompt laser for diabetic macular edema. Ophthalmology 117: 1064-1077.

25. Ness T, Feltgen N, Agostini H, Böhringer D, Lubrich B (2010) Toxic vitreitis outbreak after intravitreal injection. Retina 30: 332-338.

26. Fong DS, Custis P, Howes J, Hsu JW (2010) Intravitreal bevacizumab and ranibizumab for age-related macular degeneration a multicenter, retrospective study. Ophthalmology 117: 298-302.

27. Rasmussen A, Bloch SB, Fuchs J, Hansen LH, Larsen M, et al. (2013) A 4-year longitudinal study of 555 patients treated with ranibizumab for neovascular age-related macular degeneration. Ophthalmology 120: 26302636.

28. Brown DM, Nguyen QD, Marcus DM, Boyer DS, Patel S, et al. (2013) Long term outcomes of ranibizumab therapy for diabetic macular edema: the 36-month results from two phase III trials: RISE and RIDE. Ophthalmology 120: 2013-2322.

29. Diago T, McCannel CA, Bakri SJ, Pulido JS, Edwards AO, et al. (2009) Infectious endophthalmitis after intravitreal injection of antiangiogenic agents. Retina 29: 601-605.

30. Driscoll SJ, Klein KS, Walsh M, et al. (2009) Multicenter study of endophthalmitis after intravitreal injection of anti-vascular endothelial growth factor agents. Invest Ophthalmol Vis Sci 50: E-Abstract 6185.

31. Speaker MG, Milch FA, Shah MK, Eisner W, Kreiswirth BN (1991) Role of external bacterial flora in pathogenesis of acute postoperative endophthalimitis. Ophthalmology 98: 639-649.

32. Schwartz SG, Flynn HW, Scott IU (2009) Endophthalmitis after intravitreal injections. Expert Opin Pharmacother 10: 2119-2126.

33. Roth DB, Flynn HW Jr (2008) Distinguishing between infectious and noninfectious endophthalmitis after intravitreal triamcinolone injection. Am J Ophthalmol 146: 346-347.

34. Abell RG, Kerr NM, Allen P, Vote BJ (2012) Intravitreal injections: is there benefit for a theatre setting? Br J Ophthalmol 96: 1474-1478.

35. Bakri SJ, Risco M, Edwards AO, Pulido JS (2009) Bilateral simultaneous intravitreal injections in the office setting. Am J Ophthalmol 148: 66-69.

36. Fineman MS, Hsu J, Spirn MJ, Kaiser RS (2013) Bimanual assisted eyelid retraction technique for intravitreal injections. Retina 33: 1968-1970.

37. Aiello LP, Brucker AJ, Chang S, Cunningham ET Jr, D'Amico DJ, et al. (2004) Evolving guidelines for intravitreal injections. Retina 24: S3-S19.

38. Storey P, Dollin M, Pitcher J, Reddy S, Vojtko J, et al. (2014) The role of topical antibiotic prophylaxis to prevent endophthalmitis after intravitreal injection. Ophthalmology 121: 283-289.

39. Park Y, Kim KS, Park YH (2013) Acute endophthalmitis after intravitrea injection and preventive effect of preoperative topical antibiotics. J Ocul Pharmacol Ther 29: 900-905.

40. Speaker MG, Menikoff JA (1991) Prophylaxis of endophthalmitis with povidone-iodine. Ophthalmology 98: 1769-1775.

41. Scott IU, Flynn HW (2007) The role of topical antibiotic prophylaxis for intravitreal injections. Arch Ophthalmol 125: 974-976. 